\title{
A few thoughts about catalyzing transcription
}

\section{"From what we know at present, we have to expect that essentially all co-activators or co-repressors will either be enzymes, proteins associated with enzymes or proteins able to read the product of an enzymatic reaction."}

This editorial could be a message to the scientist who has just discovered that his favorite transcription factor interacts with a histone acetylase and who is about to publish that his field is now entering the era of epigenetics.

Only 20 years ago, the field of transcription was all about factors interacting with one another, and much of the research effort was aimed at identifying DNA-binding proteins and cis-control elements on the DNA (if nostalgic, see [1]). The logic behind this approach was that specific transcription factors brought to a promoter would in turn interact with general transcription factors and favor recruitment of the preinitiation complex and the RNA polymerase II (RNAPII). The problem with this way of thinking was that the expected interactions between the regulatory domain of the specific transcription factors and the preinitiation complex were convincingly visualized only in very rare cases.

Then came the co-activators like GCN5 and p300, and the co-repressors like Sin3a. These proteins were binding to the regulatory domain of the transcription factors and were essential for their activity. At first, the approach to study them remained the same as for the transcription factors: everyone (including me) was looking for interactions between this additional layer of regulators and the preinitiation complex. Again, this gave a lot of disappointing negative results.

Then the big breakthrough came from the laboratory of David Allis. Looking for histone acetylases in Tetrahymena, this laboratory cloned a protein with homology to GCN5. This finding made the first direct link between histone acetylation and gene activation [2]. It inferred that co-activators could be enzymes modifying the chromatin structure.

The involvement of enzymatic activities in regulation of transcription was not an entirely new idea. In fact, at the time, it was already known that chromatin remodeling machineries such as the SWI-SNF complex could function as transcription regulators. However, the link between these machineries and other types of co-regulators was not defined.

From what we know at present, we have to expect that essentially all co-activators or corepressors will either be enzymes, proteins associated with enzymes or proteins able to read the product of an enzymatic reaction. The corollary to this is that transcriptional activation or repression is most likely not about interactions, at least not directly, but rather about enzymatic activities modifying proteins present at the promoter.

\section{"In fact, there is probably no transcriptional activation without histone acetylation and inversely, repression is very systematically associated with histone deacetylation."}

The substrate of many of these enzymes is the chromatin, some using the energy from ATP hydrolysis to transiently dissociate the DNA from the nucleosome, while others covalently modify the histones.

Histone modifications can again be put into two categories, some functioning as flags binding regulatory proteins, while others directly affect the chromatin density. This later category of modifications include acetylation and phosphorylation, and will change either the charge or the folding of the histone tails, with direct consequences on the contacts established between histones and DNA, and possibly between neighboring nucleosomes (for a review see, for example, [3]).

These particular modifications are very likely to be among the end points of transcriptional regulation. In fact, there is probably no transcriptional activation without histone acetylation and inversely, repression is very systematically associated with histone deacetylation.

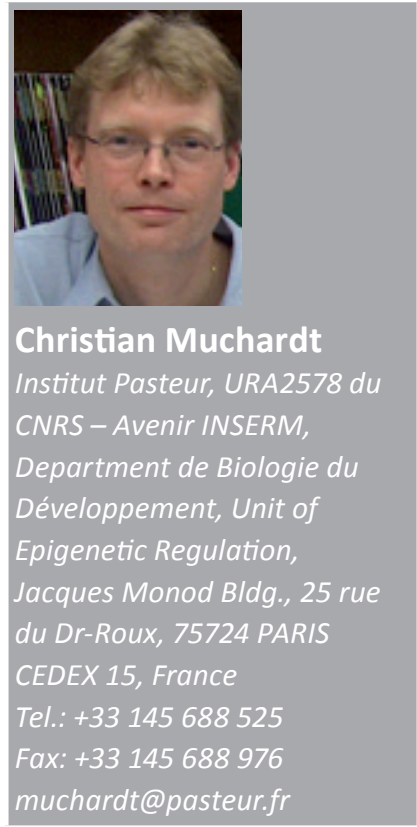

\section{muchardt@pasteur.fr}


However, modification of the histones is not the whole story. It is very likely that proteins carrying enzymatic activities at promoters will modify one another, the best example of this probably being the kinases modifying the RNAPII at the level of its C-terminal domain (CTD) [4]. It must here be noted that, until very recently, it was well accepted that the final aim of the enzymatic activities involved in transcription was to favor or to prevent recruitment of the RNAPII. This hypothesis may be weakened at the base as emerging chromatin immunoprecipitation-sequencing data now demonstrates that RNAPII is present on a large fraction of inactive promoters [5]. This suggests that the limiting step for transcriptional initiation is not RNAPII recruitment but rather phosphorylation of the CTD, a modification correlated with RNAPII promoter escape (for a review, see [6]). Subsidiarily, it also suggests that chromatin is not in itself an obstacle for RNAPII recruitment.

"This means that transcriptional regulation is likely to result from a delicate equilibrium between opposing enzymatic activities ... and is not an all-or-nothing process."

In order to complete the picture, it must finally be mentioned that depletion of a repressor very generally results in transcriptional reactivation of its target promoters. This means that transcriptional regulation is likely to result from a delicate equilibrium between opposing enzymatic activities - kinases and phosphatases, acetylases and deacetylases, methylases and demethylases - and is not an all-or-nothing process.

The conclusion from this is that, on a promoter that is off, the sum of the enzymatic activities that are present results in deacetylation of the histones and dephosphorylation of the RNAPII CTD, while on a promoter that is on, the situation is the opposite with histone acetylation and RNAPII CTD phosphorylation, the switch in itself being dictated by activation of signal transduction pathways or synthesis/degradation of factors, in connection with cellular differentiation.

Whether it is necessary to talk about epigenetic in transcriptional regulation will depend on the definition that one gives to this word, but it certainly is a process where enzymes add information on top of the DNA to change the transcriptional status of the gene. At any rate, it is clear that one should not be surprised to find that his favorite promoter is acetylated during transcriptional activation, or to discover that his favorite transcriptional activator interacts with a histone acetylase, and this is true in any field of research, whether it is development, immunity, host-pathogen relations and so on. Therefore, if finding that a transcription activator is interacting with a histone acetylase is not really exciting after all, then what is? What are the new frontiers in understanding transcriptional regulation?

\section{"As the histone tails are highly charged, it is tempting to speculate that they may interact with ... RNAs."}

Many of the unsolved issues are of course about mechanism. What is acetylation of histone tails really doing to the chromatin structure? What are the remodeling complexes really doing to the nucleosomes? How is CTD phosphorylation linked to the processivity of the RNAPII?

The role of RNA is also an interesting issue. Many promoters are transcribed and produce small RNAs [7-10]. As the histone tails are highly charged, it is tempting to speculate that they may interact with these RNAs, much in the same way ribosomal proteins interact with, and structure, ribosomal proteins [11]. This may in turn create new docking sites for regulatory proteins.

Alternatively, promoter RNAs may be involved in RNAi-mediated transcriptional repression. Homing of histone methylases via short RNAs able to locate their site of transcription by hybridizing with the neosynthesized transcript has been described in yeast (for a recent review, see [12]). In higher eukaryotes, the existence of such a mechanism is suspected but not yet demonstrated.

\section{Financial \& competing interests disclosure}

The author has no relevant affiliations or financial involvement with any organization or entity with a financial interest in or financial conflict with the subject matter or materials discussed in the manuscript. This includes employment, consultancies, honoraria, stock ownership or options, expert testimony, grants or patents received or pending, or royalties.

No writing assistance was utilized in the production of this manuscript. 


\section{Bibliography}

1 Ptashne M, Gann AA: Activators and targets. Nature 346(6282), 329-231 (1990).

2 Brownell JE, Zhou J, Ranalli T et al:: Tetrahymena histone acetyltransferase A: a homolog to yeast Gcn5p linking histone acetylation to gene activation. Cell 84(6), 843-851 (1996).

3 Ruthenburg AJ, Li H, Patel DJ, Allis CD: Multivalent engagement of chromatin modifications by linked binding modules. Nat. Rev. Mol. Cell Biol. 8(12), 983-994 (2007).

4 Egloff S, Murphy S: Cracking the RNA polymerase II CTD code. Trends Genet. 24(6), 280-288 (2008).
5 Muse GW, Gilchrist DA, Nechaev S et al.: RNA polymerase is poised for activation across the genome. Nat. Genet. 39(12), 1507-1511 (2007).

6 Perales R, Bentley D: 'Cotranscriptionality': the transcription elongation complex as a nexus for nuclear transactions. Mol. Cell 36(2), 178-191 (2009).

7 Kapranov P, Cheng J, Dike S et al.: RNA maps reveal new RNA classes and a possible function for pervasive transcription. Science 316(5830), 1484-1488 (2007).

8 Seila AC, Calabrese JM, Levine SS et al.: Divergent transcription from active promoters. Science 322(5909), 1849-1851 (2008).
9 Core LJ, Waterfall JJ, Lis JT: Nascent RNA sequencing reveals widespread pausing and divergent initiation at human promoters. Science 322(5909), 1845-1848 (2008).

10 Preker P, Nielsen J, Kammler S et al.: RNA exosome depletion reveals transcription upstream of active human promoters. Science 322(5909), 1851-1854 (2008).

11 Brodersen DE, Clemons WM Jr, Carter AP, Wimberly BT, Ramakrishnan V: Crystal structure of the $30 \mathrm{~S}$ ribosomal subunit from Thermus thermophilus: structure of the proteins and their interactions with $16 \mathrm{~S}$ RNA. J. Mol. Biol. 316(3), 725-768 (2002).

12 Verdel A, Vavasseur A, Le Gorrec M, Touat-Todeschini L: Common themes in siRNA-mediated epigenetic silencing pathways. Int. J. Dev. Biol. 53(2-3), 245-257 (2009). 